\title{
DISTRIBUCIÓN Y PREFERENCIAS CLIMÁTICAS DE Sobralia uribei (ORCHIDACEAE): UNA ESPECIE ENDÉMICA DE SANTANDER, COLOMBIA
}

\author{
Miguel Macgayver Bonilla Morales ${ }^{1}$ \\ Oscar Iban Hernández Castañeda ${ }^{2}$ \\ Adriana Carolina Aguirre Morales ${ }^{3}$
}

Recibido el 13 de marzo de 2015, aprobado el 31 de agosto de 2015 y actualizado el 18 de mayo de 2016

DOI: 10.17151/luaz.2016.43.7

\section{RESUMEN}

En Colombia alrededor del 35\% de las especies registradas son endémicas y, en algunos casos, sólo son conocidas por el espécimen o localidad tipo. No obstante, el avance de la frontera agrícola, la deforestación, la minería y otras prácticas antropogénicas, son las principales causas de su extinción. Los estudios realizados en Santander, desde la decada del sesenta, determinaron que Sobralia uribei, especie endémica de este departamento, es conocida en una localidad del municipio de Piedecuesta. El objetivo de esta investigación fue conocer la diversidad intraespecífica, distribución y estado actual de conservación de $\boldsymbol{S}$. uribei. Por esta razón, se revisaron los herbarios locales y se realizaron salidas de campo en el departamento de Santander. Los datos obtenidos fueron procesados para análisis morfológicos y biogeográficos mediante el programa DIVA-GIS. Después de 54 años, se registra una nueva localidad en el municipio de Contratación, a una distancia en línea recta de 84 km, y un promedio de 1500-2000 msnm. Los caracteres morfológicos diferenciales fueron la altura de la planta (1-3 $\mathrm{m}$ ), el color del ápice del labelo (blanco o lila), y el número de flores por racimo (911vs 7-9). La distribución potencial se concentró en los municipios de El Carmen, San Vicente de Chucurí, Hato, Galán y Zapatoca, entre los 1500 a 2600 msnm, y no donde fue colectada. La temperatura de las subpoblaciones no presenta diferencias significativas, y los inicios de la precipitación de enero a abril permiten la floración de la especie. El estatus de conservación de $\boldsymbol{S}$. uribei se defiene en la categoría de Peligro Crítico (CR) según los criterios de riesgo propuestos por la UICN. Actualmente, la intervención antrópica es el factor principal que puede causar la extinción de las subpoblaciones existentes, por lo que estrategias de conservación in situ y ex situ son urgentes y necesarias. 


\title{
PALABRAS CLAVE
}

Biogeografía, conservación, orquídea, Sobralieae.

\section{DISTRIBUTION AND CLIMATE PREFERENCES Sobralia uribei (ORCHIDACEAE): AN ENDEMIC SPECIES FROM SANTANDER, COLOMBIA}

\begin{abstract}
In Colombia, about $35 \%$ of the recorded species are endemic and in some cases are only known specimen type or location. However, the advance of the agricultural frontier, deforestation, mining and other anthropogenic practices are the main causes of extinction. Studies in Santander, from the sixties, determined that Sobralia uribei, endemic to this department, is known in a locality in the municipality of Piedecuesta. The objective of this research was to study the intraspecific diversity, distribution and state of conservation of S. uribei. For this reason, local herbal reviewed and conducted field trips in the Department of Santander. The data obtained were processed for morphological and biogeographic analysis using DIVA-GIS program. After 54 years, a new location is recorded in the municipality of Trade, a straight line distance of $84 \mathrm{~km}$, and an average of 1500 to $2000 \mathrm{~m}$. The differential morphological characters were plant height (1-3 m), the apex of the lip color (white or purple), and the number of flowers per cluster (9-11 vs 7-9). The potential distribution is concentrated in the towns of El Carmen, San Vicente de Chucurí, Hato, Galán and Zapatoca, between 1500-2600 m, and not where it was collected. The temperature of subpopulations no significant differences and the beginning of the precipitation from January to April, allowing the flowering of the species. The conservation status of $\mathbf{S}$. uribei is defiene in the category of Critically Endangered (CR) according to risk criteria proposed by IUCN. Currently, human intervention is the main factor that may cause the extinction of existing subpopulations, so conservation strategies in situ and ex situ, are urgent and necessary.
\end{abstract}

\section{KEY WORDS}

Biogeography, conservation, orchid, Sobralieae. 


\section{INTRODUCCIÓN}

Las familias Orchidaceae y Asteraceae representan el mayor número de especies vegetales en el mundo (Simpson, 2010), incluyendo la cuarta parte de las 250.000 especies de angiospermas registradas (Chase et al., 2015). En Colombia existen cerca de 4.100 especies de orquídeas agrupadas en 232 géneros (Ordóñez y Montes-Pulido, 2013). El 87,2\% de las especies están en la región Andina, seguida por las regiones Pacífica y Amazónica cada una con un 10,6\%, el Caribe con el $5 \%$, y la Orinoquía con el $4 \%$ (Sarmiento, 2007). A pesar de que Colombia es uno de los países con mayor diversidad de especies de orquídeas, su estudio se ha limitado al registro de especies nuevas o inventarios, y no al conocimiento biogeográfico y de conservación (Bonilla, Gallego y Aguirre, 2014; Betancur et al., 2015).

La creciente actividad antrópica ha generado efectos ambientales que amenazan con la distribución y las áreas de conservación de especies silvestres y endémicas del país, como las orquídeas (Camargo y Delgado, 2006; Bonilla, Yepes y Otero, 2014; Betancur et al., 2015). Las principales causas de la pérdida de biodiversidad de los Andes se encuentran asociadas a cambios de hábitat, sobreexplotación, cambio climático y polución, que se relacionan con la ocupación actual de los cultivos agrícolas y pecuarios (Bellard et al., 2012; Pereira, Navarro y Santos, 2012). La mitad de la diversidad biológica registrada en los Andes es endémica (Myers et al., 2000), pues su estrecha relación al suelo, precipitación, temperatura, variabilidad de los nichos ecológicos específicos (Herzog et al., 2012), la hace una región con caracteríticas exclusivas para el crecimiento de microorganismos, hongos, fauna y flora específica. De tal manera, la región Andina por su alto grado de diversidad, endemismo y amenaza, es considerada un hotspots o punto caliente (Myers et al., 2000).

Sobralia uribei P. Ortiz es una orquídea terrestre que crece en la biorregión andina del departamento de Santander (Colombia), y es considerada como una especie endémica, conocida solamente por su localidad tipo (Mesa de los Santos, Piedecuesta). En 1962 Lorenzo Uribe hizo la primera colecta y descripción de la planta, sin embargo, fue el Padre Pedro Ortiz quien la reportó como especie nueva para la ciencia en 1994 (Ortiz, 1994). Estudios posteriores de Camargo y Delgado (2006) de la orquideoflora del municipio de Piedecuesta (Santander), la reportan para el mismo sitio. No obstante, este lugar ha sido alterado a causa del saqueo de los bosques y de la expansión ganadera, por lo que la especie enfrenta un alto 
grado de amenaza. La presente investigación tuvo como objetivo establecer una descripción morfológica apoyada en ilustración científica, determinar las zonas de ocupación actual, así como de distribución potencial, las preferencias climáticas y el estatus de conservación de Sobralia uribei.

\section{MATERIALES Y MÉTODOS}

\section{Área de estudio}

El estudio se realizó en el departamento de Santander (Colombia) al nororiente de los Andes (05 $42^{\prime} 34^{\prime \prime} \mathrm{N}$ y $08^{\circ} 07^{\prime} 32^{\prime \prime}$ de longitud Oeste), el cual abarca una extensión de $30.537 \mathrm{~km} 2$, un rango altitudinal entre 250 y $4000 \mathrm{msnm}$ y una temperatura que oscila entre $6-30^{\circ} \mathrm{C}$.

\section{Herbarios y literatura}

Se visitaron y revisaron colecciones botánicas de los herbarios nacionales COL, CDMB y UIS. Además, se consultaron herbarios internacionales en línea como MO, KEW y sitios web como The Plant List y el GBIF. Se revisó literatura científica de orquídeas que relacionó el departamento de Santander de: Ortiz (1994), Ortiz y Uribe $(2007,2014)$ y López (2011). Para la identificación taxonómica de la especie se tuvo la validación de la publicación tipo y la asesoría del experto en el grupo.

\section{Fase de campo}

Durante el período comprendido entre los años 2013 al 2015, se realizaron viajes principalmente a los municipios de Bucaramanga, Contratación, Floridablanca, Girón, Matanza, Charta, Piedecuesta, Tona, y se exploraron bosques, senderos y caminos de carretera. Cuando se encontró la especie se hizo el registro fotográfico, la antoteca (colecta de la flor en líquido), toma de datos de georreferenciación y colecta de espécimen de herbario con la finalidad de su posterior identificación. 


\section{Morfología}

Se tomaron los datos morfológicos que relacionan hábito, tallo, hojas, inflorescencia y flor, para la descripción y verificación de la especie. Además, con base en los registros fotográficos, flores en alcohol y datos morfológicos se procedió a realizar la ilustración científica, para complementar el dibujo tipo de Ortiz (1994) y determinar las relaciones con las especies más afines.

\section{Distribución y preferencias climáticas}

Los datos fueron analizados con el programa DIVA-GIS@ versión 7.5 con la finalidad de establecer la distribución mediante puntos en el mapa de Santander con capa de Elevación para Surámerica. Seguido, se usó el modelo climático de ocurrencias de especies del paquete WorldClim en DIVA-GIS@ para determinar las preferencias climáticas generando un mapa de distribución potencial (Hijmans et al., 2005), y para extraer las 19 variables climáticas de las capas de Bioclim que relacionan los componentes de temperatura y precipitación para su análisis. Los mapas se editaron con el mismo software siguiendo lo propuesto por Hijmans, Guarino y Mathur (2012).

\section{Conservación}

En cuanto a los aspectos de conservación se tuvieron en cuenta los criterios planteados por la IUCN (2003) y los asociados por Calderón-Sáenz (2006) para establecer el estatus de conservación de orquídeas colombianas. Posteriormente, se complementó para la evaluación de la especie el Área de Extensión (EOO) y Ocupación (AOO) calculados con la plataforma GeoCAT (Bachman et al., 2011) con la finalidad de establecer la categoría de Conservación del taxón en estudio.

\section{RESULTADOS Y DISCUSIÓN}

\section{Relaciones morfológicas}

Para Colombia se registran 47 especies del género Sobralia, de las cuales 19 son endémicas, incluida la especie en estudio $\mathbf{S}$. uribei (Figura 1). Sin embargo, Ortiz y Uribe (2007) registran 42 spp., excluyendo a las especies $\mathbf{S}$. anceps Schltr., S. antioquiensisSchltr., S. chrysantha Lindl., S. decora Bateman, S. 
hoppii Schltr., S. lacerata Dressler \& Pupulin, S. madisonii Dodson, S. malmquistiana Schltr., S. paludosa Linden, S. paradisiaca Rchb. f., S. piedrahitae $\mathrm{P}$. Ortiz, S. rigidissima Linden ex Rchb. f., y S. schultzei Schltr. Además de las especies reportadas por Ortiz y Uribe (2007), S. suaveolensRchb. f. es sinónimo de S. bletiae Rchb. f. y junto con S. ciliata (C. Presl) C. Schweinf. ex Foldats son no conocidas de espécimen de herbario para Colombia, mientras que S. sessilisLindl. y S. fenzliana Rchb. f. son sinónimos de S. decora Bateman. El género Sobralia que hace parte de la subfamilia Epidendroideae, tribu Sobraliae posee cerca de 100 especies (Pridgeon, 2005; Neubig et al., 2011), esto permite concluir que en Colombia se encuentra aproximadamente el $50 \%$ de las especies que constituyen el grupo. No obstante, Chase et al. (2015) en trabajo que involucra la actualización de la clasificación de la familia Orchidaceae, establecen 142 especies para el taxón.
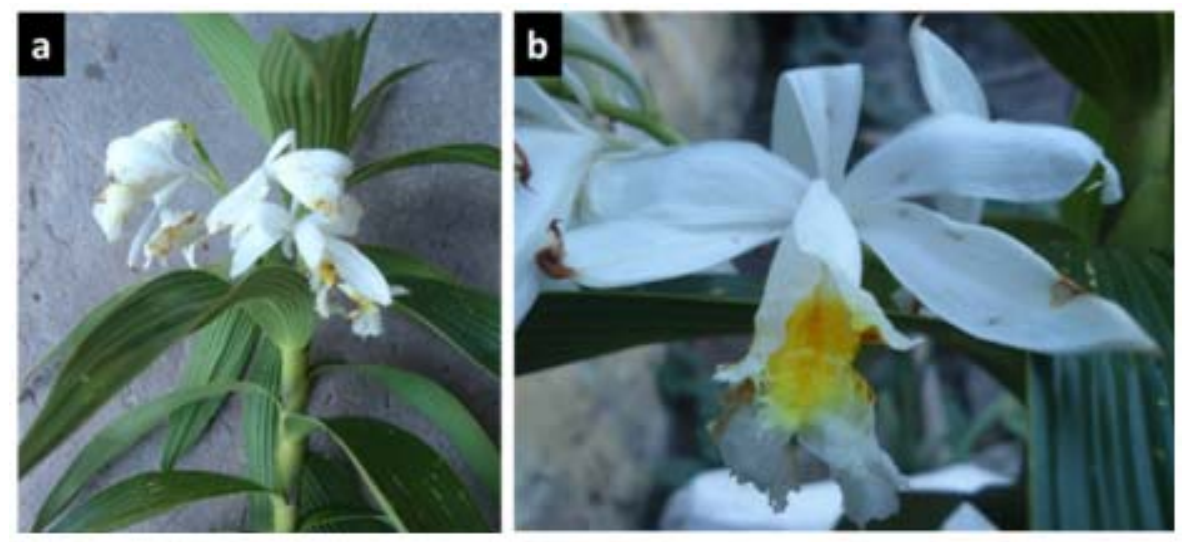

Fuente: los autores.

Figura 1. Sobralia uribei. a) planta con la inflorescencia, b) flor.

Los caracteres diagnósticos del género Sobralia son: flores grandes, rápida senescencia (en su mayoría un día), sépalos y pétalos laterales similares, labelo fimbriado o crestado que rodea la columna con dos carinas en la base donde descansa la columna, antera operculada con polinios harinosos que forman cuatro bandas y estigma emergente (Pridgeon, 2005). La especie Sobralia uribei es afín a S. roezlii, pero se diferencia porque $S$. uriberi tiene las hojas oblongas y acuminadas, sépalos laterales no falcados, pétalos oblongo-elípticos y labelo abovado (Ortiz, 1994). La ilustración realizada por Ortiz (1994) de la especie tipo no establece detalles específicos que permitan visualizar y comprender mejor la especie desde sus caracteres morfométricos, de acuerdo a lo que se plantea en la descripción, pues no solo carece de una flor de perfil, columna, polinios o antera, 
sino que además no se resaltan caracteres de importancia taxonómica en el dibujo como los detalles de las carinas. Por esta razón, se realizó una nueva ilustración que incluyera las partes faltantes en la ilustración tipo y se profundizó en los trazos. Se utilizó la técnica de puntillismo para demostrar los atributos que diferencian a la especie de aquellas con las que presenta relaciones interespecíficas (Figura 2).

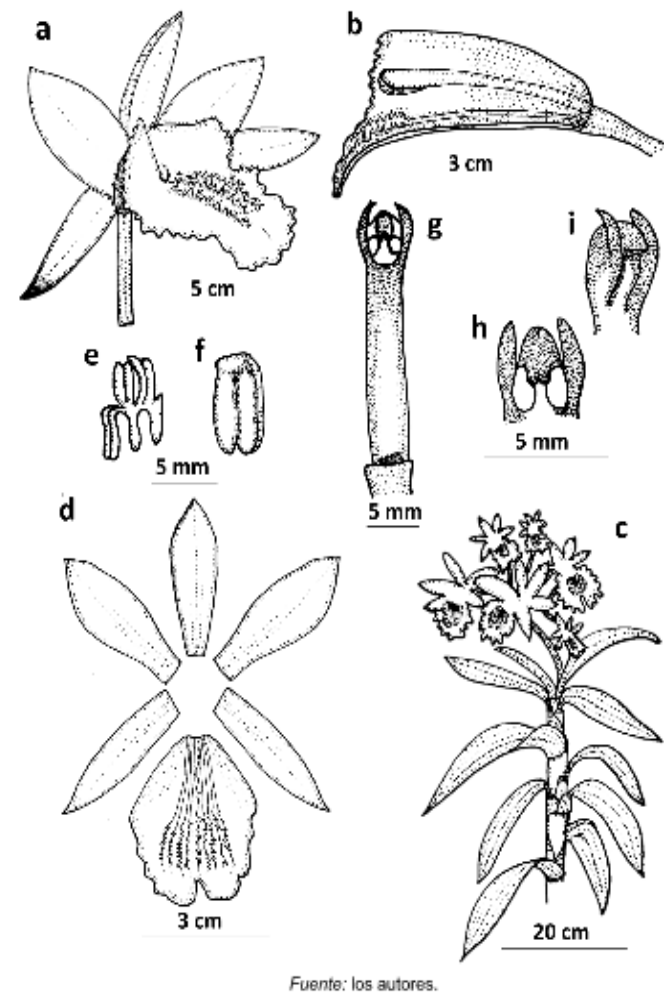

Figura 2. Ilustración de Sobralia uribei. a) perfil flor, b) corte longitudinal del labelo, c) planta, d) sépalo dorsal, sépalos y pétalos laterales y labelo extendidos, e) polinios, $f$ ) antera, g) columna en posición ventral, h) perfil del ápice de la columna (antera y estigmas), i) perfil del ápice de la columna.

La Tabla 1 presenta las características morfológicas de la subpoblación encontrada en el municipio de Contratación (Santander). Las principales diferencias se asocian a la altura de la planta $(1 \mathrm{~m})$, coloración del ápice del labelo (blanco) y una inflorescencia axilar en racimo con 7-9 flores. Este último carácter concuerda con la descripción de Ortiz (1994), de que puede tener una o más inflorescencias con numerosas flores. En comparación, a la descripción de la especie tipo realizada por Ortiz (1994) la altura va hasta 2 m, mientras Camargo y Delgado (2006) definen que puede medir de 2,5-3 m de altura, de la misma planta encontrada en la localidad tipo. Los pétalos y sépalos blancos con ápice ligeramente teñidos de lila, 
labelo con coloración blanca y amarilla en el centro con ligera tinción de lila en el ápice (Ortiz, 1994; Camargo y Delgado, 2006). En la galería de fotos de orquídeas presentada por Ortiz y Uribe (2007) se muestran imágenes con labelo de color blanco, amarillo en el centro y lila en el ápice de la especie tipo en Piedecuesta, que corroboran la información de Ortiz (1994) y Camargo y Delgado (2006). La descripción de los caracteres morfológicos florales y vegetativos concuerda con estos autores, y aunque existe variación del tamaño de la flor, esto probablemente sea debido al ambiente de la localidad de procedencia.

Tabla 1. Caracteres morfológicos de Sobralia uribei en Contratación (Santander)

\begin{tabular}{|c|c|c|c|c|c|}
\hline $\begin{array}{c}\text { Carácter } \\
\text { morfológico }\end{array}$ & Posición/Forma & $\begin{array}{l}\text { Largo } \\
(\mathrm{cm})\end{array}$ & $\begin{array}{l}\text { Ancho } \\
(\mathrm{cm})\end{array}$ & Apice & Nota \\
\hline Tallo & Arundíneo & 100 & & & $\begin{array}{c}\text { 1,5 Diámetro, } \\
\text { terete/Hábito } \\
\text { terrestre }\end{array}$ \\
\hline Hojas & HelicoidaVOblonga & $23,7-24,9$ & $3,2-3,6$ & Acuminada & $\begin{array}{c}\text { Envainadora, } \\
\text { plicadas, } \\
11-13 \\
\text { Nervaduras }\end{array}$ \\
\hline Inflorescencia & Axial-péndula & & & & 7 Flores \\
\hline Bráctea & Triangular & $0,9-1$ & $0,35 \cdot 0,4$ & Agudo & Envainadora \\
\hline Ovario & Terete & $2,2-2,8$ & $0,3-0,35$ & & Sésil \\
\hline Sépalo dorsal & /Oblongo-elíptico & $4,5-4,6$ & $1,6-1,8$ & Oblicuo & $\begin{array}{c}\text { Blanco, margen } \\
\text { entero }\end{array}$ \\
\hline $\begin{array}{l}\text { Sepalos } \\
\text { laterales }\end{array}$ & /Oblongo-elípticos & $4,9-5$ & $1,2-1,3$ & Oblicuo & $\begin{array}{c}\text { Blanco, margen } \\
\text { entero }\end{array}$ \\
\hline $\begin{array}{l}\text { Petalos } \\
\text { laterales }\end{array}$ & /Oblongo-elipticos & $4,8-4,9$ & $1,1-1,2$ & Oblicuo & Blanco \\
\hline Labelo & /Obovado & $4,6-4,8$ & $2,9-3$ & Bilobulado & Blanco \\
\hline $\begin{array}{l}\text { Labelo carinas } \\
\text { o quillas }\end{array}$ & & $2,5-2,7$ & & & 7 , setigero \\
\hline Columna & Triangular/Recta & $1,5-1,6$ & & & Apice alado \\
\hline Estigma & Ventral/Bilobulada & 0,2 & 0,3 & & \\
\hline Antera & Ventral/Incumbente & 0,65 & 0,3 & & Bilocular, blanca \\
\hline Polinio & Apice & 0,3 & & & $\begin{array}{c}8 \text { (4 en cada } \\
\text { lóculo), sectile }\end{array}$ \\
\hline
\end{tabular}

Dentro de las especies que presentan caracteres morfológicos afines con $\mathbf{S}$. uribei estánSobralia mutisii P. Ortiz, S. dichotoma Ruiz \& Pav., S. roezlii Rchb. f., y S. catleya Rchb. f. (Ortiz y Uribe, 2007). Las principales diferencias con S. mutisii son altura de la planta de 4-5 m versus 1-2,5 $\mathrm{m}$ en $\mathbf{S}$. uribei, hojas lanceolado-acuminadas vs oblongo-acuminadas en $\mathbf{S}$. uribei, sépalos y pétalos elípticos vs oblongo-elípticos, y labelo ovalado vs obovado en S. uribei (Ortiz, 2004). Por otro lado, se encuentra la especie Sobralia dichotoma, conocida por 
mucho tiempo como la especie tipo del género, aunque por normatividad del Código Internacional de Nomenclatura Botánica existe una propuesta de conservación del tipo que la valida (Dressler et al., 2011). Su principal disimilitud con S. uribei es la coloración de las flores púrpura o rosadas vs blancas (Carnevali et al., 2003), aunque posee otros caracteres morfométricos no similares, como lóbulos del labelo y carinas. Además, esta especie junto con S. catleya y $\mathbf{S}$. roezlii, se diferencia por la base del labelo y su forma de cobertura a la columna (Kolanowska y Szlachetko, 2013).

\section{Distribución y conservación}

Sobralia uribei es una especie endémica de Colombia con una densa subpoblación en el municipio de Piedecuesta, localidad la Mesa de los Santos (Santander), considerado como el único lugar de registro en el mundo (Camargo y Delgado, 2006). En esta localidad fue encontrado el espécimen tipo en 1962 por el Padre Lorenzo Uribe, posteriormente (20 años después) el Padre Ortiz la halla en la misma localidad y la describe como especie nueva en honor a su descubridor (Ortiz, 1994). La extensión que tiene Colombia, su biodiversidad y conformación de paisajes, lo hace un país escasamente explorado y que ofrece grandes hallazgos. La presente investigación registra la especie en la vereda San Juanera del municipio de Contratación (Santander), ubicada al suroriente del departamento a $84 \mathrm{~km}$ en línea recta aproximada a Mesa de los Santos, donde se encontró la localidad tipo de la especie en la Cordillera Oriental de los Andes (Figura 3). 


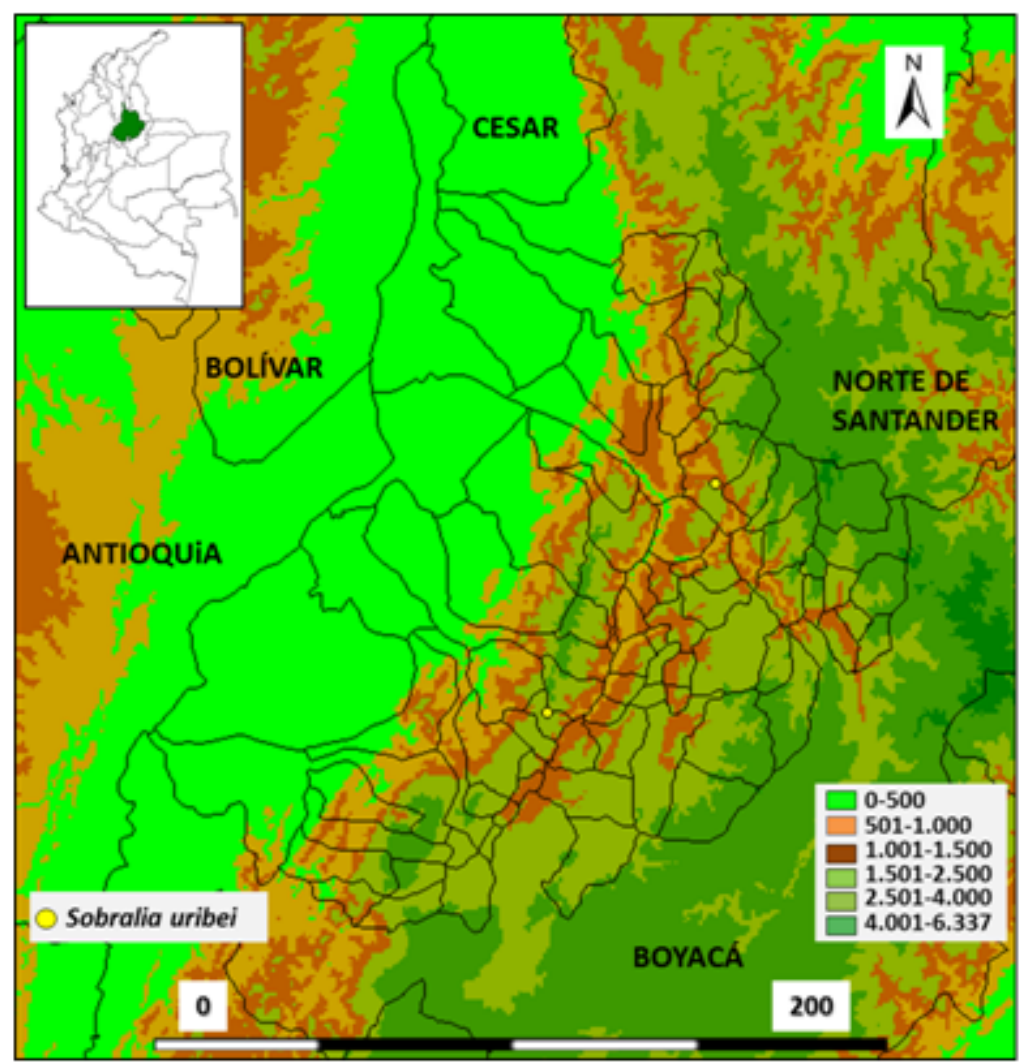

Fuente: los sutores.

Figura 3. Distribución de Sobralia uribei, Santander, Colombia.

Por el área de extensión y el área de ocupación que tiene la especie, y sus subpoblaciones solo en dos localidades con espacios restringidos, ésta puede ser definida como una especie que enfrenta algún tipo de amenaza, y dadas las caraterísticas mencionadas se puede catalogar bajo el criterio de "En Peligro Crítico" (CR) (IUCN, 2003). De acuerdo a lo mencionado por Calderón-Sáenz (2006) se determina que $S$. uribei es una especie amenzada, por cumplir con los criterios $\mathrm{A} 1 \mathrm{a}, \mathrm{A} 2 \mathrm{ac}$ y $\mathrm{A} 3 \mathrm{c}$ que relacionan la reducción de las subpoblaciones de la localidad tipo por actividades antrópicas actuales (Camargo y Delgado, 2006), criterio B1a y B2a, sobre el EOO y AOO como el número de localidades (2), y el criterio $\mathrm{C} 1$ por el número de individuos para cada una de las subpoblaciones.

\section{Relaciones climáticas}

La Figura 4 establece la distribución potencial para S. uribei con mayor concentración en los municipios de El Carmen y San Vicente de Chucurí, Hato, Galán y Zapatoca (círculo azul grande), entre los 1500 a 2600 msnm, y no donde 
ha sido colectada (Figura 3). Es conocido que efectos antropogénicos y efectos del cambio climático en el presente y en el futuro, relacionados con la temperatura y la precipitación, afectarán especies nativas como endémicas de los Andes (Herzog et al., 2012). Dentro de estas especies vulnerables se registran las subpoblaciones de $\mathbf{S}$. uribei, que con base en la modelación de distribución potencial, serán afectadas. No obstante, se registran otros municipios poco conocidos donde el nicho ecológicos es viable para que se establezcan o donde pueden existir otras subpoblaciones.

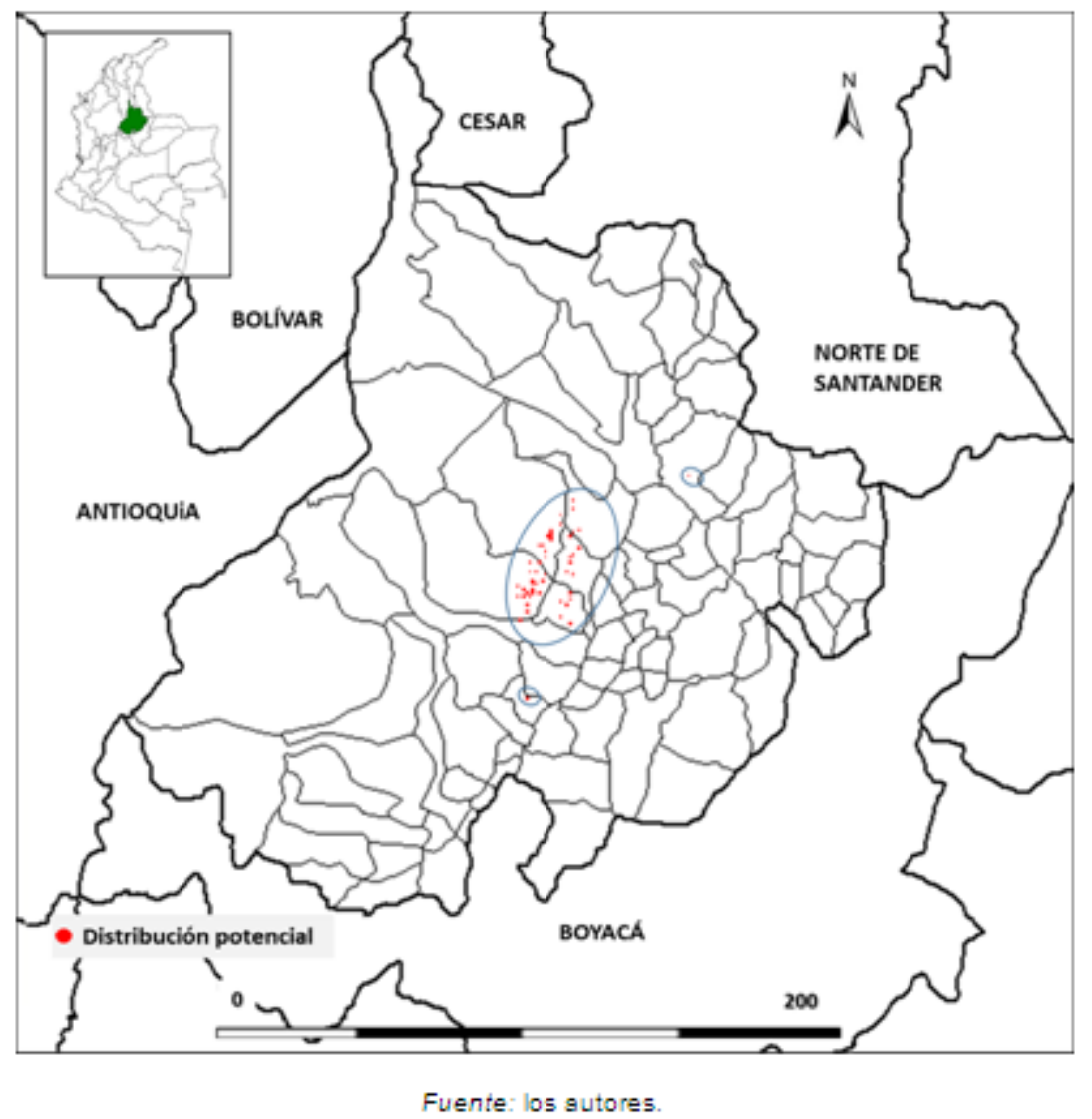

Figura 4. Distribución potencial de S. uribei, Santander, Colombia.

Sumado a lo anterior, se deben realizar salidas de campo a las zonas de preferencia de nicho por la especie de acuerdo a lo registrado por la distribución potencial, en busca de otras subpoblaciones o sitios para su manejo y conservación (Bonilla, 2014). Cabe resaltar que los registros de las subpoblaciones in situ no se encuentran dentro de las áreas protegidas nacionales. Esto hace que la especie sea más susceptible a la desaparición de los individuos existentes, particularmente, por los cambios de hábitat que generan las 
poblaciones humanas para su asentamiento, cultivos agropecuarios, deforestación o extracción de hidrocarburos o minerales (Pereira et al., 2012).

De otro lado, la temperatura en ambas localidades (Contratación y Piedecuesta) no presenta diferencias significativas, y las precipitaciones de enero a abril permiten la floración de la especie (Figura 5). Ambas localidades presentan un patrón similar, pues se requieren precipitaciones mensuales alrededor de los $100 \mathrm{~mm}$ y que no superen los $150 \mathrm{~mm}$, para que se presente la floración de S. uribei. En Mesa de los Santos la especie se ha encontrado en flor a principios de abril (Ortiz, 1994), mientras que en Contratación se encontró en enero. No obstante, se podría considerar que el patrón de floración puede ser dos veces al año, una para finales de diciembre e inicios de enero y la segunda a finales de marzo e inicios de abril, en ambos sitios.

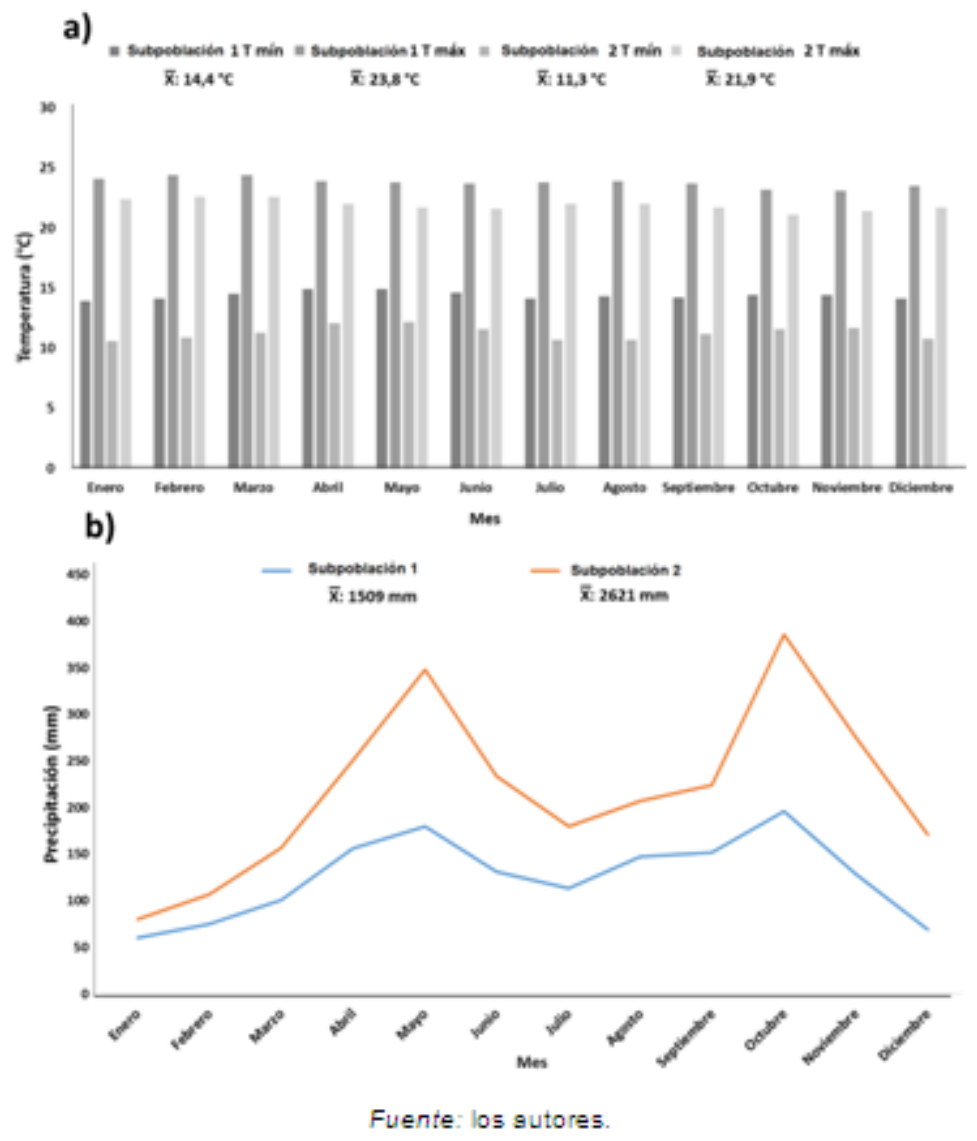

Figura 5. Precipitación y temperatura anual de los hábitats de Sobralia uribei. 
Dentro de las especies de Sobralia registradas para Santander, están S. ruckeri Linden \& Rchb. f. en el municipio de Málaga (López, 2011), S. violacea Linden ex Lindl. en la Mesa de los Santos y vía a Pamplona, S. macrophylla Rchb. f. (Ortiz, 1994; Camargo y Delgado, 2006; Martínez, Bonilla y López, 2015). Además, en Piedecuesta se encuentra S. poweliiSchltr. y en Charta S. virginalis Peeters \& Cogn. Aunque ninguna de estas especies se encuentra relacionada morfológicamente con $\mathbf{S}$. uribei, componentes como la estructura y textura del suelo donde crecen o las condiciones climáticas tienden a ser afínes, pues la floración de las especies coincide en el departamento de Santander.

\section{CONCLUSIONES}

La distribución potencial principalmente determina nichos ecológicos preferentes asociados a los municipios que no hacen parte de la localidad tipo ni del nuevo registro de sitio reportado en este trabajo, por tal motivo, salidas de campo a estos lugares son urgentes y necesarias para conocer mejor la distribución de la especie o establecer sitios de manejo de las subpoblaciones existentes para su conservación in situ.

\section{AGRADECIMIENTOS}

Los autores agradecen al jefe de bombero y senderista Uber Báez, por su apoyo y colaboración en la colecta del material vegetal. Además, a los profesores Alfonso Morales y Oscar Agudelo Varela de la Universidad de los Llanos.

\section{POTENCIAL CONFLICTO DE INTERESES}

Esta investigación no presenta conflicto de intereses. 


\section{FUENTES DE FINANCIACIÓN}

Esta investigación hace parte de las salidas y colectas del proyecto 'Taxonomía y Biogeografía de Passiflora' del grupo Horizonte Mediático de la Universidad de los Llanos, Sede Barcelona, Villavicencio, Meta.

\section{REFERENCIAS}

- Bachman, S.; Moat, J.; Hill, A.; Torre, J. y Scott, Ben. (2011). Supporting Red List threat assessments with GeoCat: geospatial conservation assessments tool. Zookeys, 150:117-126.

- Bellard, C.; Bertelsmier, C.; Leadley, P.; Thuiller, W. y Courchamp, F. (2012). Impacts of climate change on the future of bioversity. Ecology Letters, 15(4), 365377.

- Betancur, J.; Sarmiento, H.; Toro-González, L. y Valencia, J. (2015). Plan para el estudio y la conservación de orquídeas en Colombia. Universidad Nacional de Colombia, Facultad de Ciencias, Instituto de Ciencias Naturales y Ministerio de Ambiente.

- Bonilla, M. (2014). Capítulo 2: Diversidad y Biogeografía de Passiflora L. supersección Tacsonia (Passifloraceae) del trópico andino. Tesis para optar al título de maestría en Ciencias Biológicas, Universidad Nacional de Colombia, Sede Palmira.

- Bonilla, M.; Gallego, E. y Aguirre, C. (2014). Notas sobre Galeandra beyrichii Rchb. f. (Orchidaceae: Catasetinae) para Colombia. Orquideología, 31(1), 33-44.

- Bonilla, M.; Yepes, D. y Otero, J. (2014). Diversidad y distribución de Catasetum rectangulare G.F. Carr (Orchidaceae: Catasetinae). Orquideología, 31(1), 15-25.

- Calderón-Sáenz, E. (Ed.). (2006). Libro Rojo de Plantas de Colombia. Volumen 3: Orquídeas, Primera Parte. Serie Libros Rojos de Especies Amenazadas de 
Colombia. Bogotá, Colombia: Instituto Alexander von Humboldt - Ministerio de Ambiente, Vivienda y Desarrollo Territorial.

- Camargo, C. y Delgado, C. (2006). Flora Orchidaceae de la Mesa de los Santos (Piedecuesta-Santander). Trabajo de grado para optar el título de Biólogo. Universidad Industrial de Santander, Bucaramanga.

- Carnevali, G.; Ramírez-M., I.; Romero-G., A.; Vargas, C. y Foldats, E. (2003).Orchidaceae. En Berry, P.E. et al. (Eds.), Flora of the Venezuelan Guayana (pp. 200-619). Volumen 7. St. Louis: Missouri Botanical Garden.

- Chase, M.; Cameron, K.; Freudenstein, J.; Pridgeon, A.; Salazar, G.; Van Den Berg, C. y Schuiteman, A. (2015). An updated classification of Orchidaceae. Botanical Journal of the Linnean Society, 177, 151-174.

- Dressler, R.; Blanco, M.; Pupulin, F. y Neubig, K. (2011). (2019) Proposal to conserve the name Sobralia (Orchidaceae) with a conserved type. Taxon, 60(3), 907-908.

- Herzog, S.; Martínez, R.; Jorgensen, P. y Tiesen, H. (2012). Cambio climático y biodiversidad en los Andes tropicales. Inter-American institute for Global Change Research (IAI) and Scientific Committee on Problems of the Environment (SCOPE).

- Hijmans, R.; Cameron, S.; Parra, J.; Jones, P. y Jarvis, A. (2005). Very high resolution interpolated climate surfaces for global land areas. International Journal of climatology, 25, 1965-1978.

- Hijmans, R.; Guarino, L. y Mathur, P. (2012). DIVA-GIS.A geographic information system for the analysis of species distribution data. Manual, version 7.5.

- IUCN. (2003). Guidelines for application of IUCN Red List criteria at regional levels: Version 3.0. IUCN Species Survival Commission. IUCN, Gland, Switzerland and Cambridge, UK.

- Kolanowska, M. y Szlachetko, D. 2013. Orchids of the Department of Valle del Cauca (Colombia). Volume 1: Cypripediaceae, Orchidaceae (Orchidioideae, Spiranthoideae, Vanilloideae, Epidendroideae (Malaxidae, Elleantheae)). Koeltz Scientific Books. 
- López, H. (2011). Orquídeas nativas de Santander. Santander, Colombia: Gobernación de Santander.

- Myers, N.; Mittermeier, R.; Mittermeier, C.; Fonseca, G. y Kent, J. (2000). Biodiversity hotspots for conservation priorities. Nature, 403, 853-858.

- Martínez, S.; Bonilla, M. y López, H. 2015. Listado de la flora Orchidaceae de Santander y comentarios sobre sus especies endémicas. Revista Facultad de Ciencias Básicas. 11 (2),In press.

- Neubig, K.; Whitten, M.; Blanco, M.; Endara, L.; Williams, N.H. y Koehler, S. (2011).Preliminary molecular phylogenetics of sobralia and relatives (Orchidaceae: Sobralieae).Lankesteriana, 11(3), 307-317.

- Ordóñez, J. y Montes-Pulido, C. (2013). Orquideoflora de la Reserva Natural Quininí, Tibacuy, Cundinamarca, Colombia y consideraciones para su bioprospección.Orquideología, 30(1), 18-33.

- Ortiz, P. (1994). Sobrariauribei Ortiz, sp. nov. Orquideología, 19(3), 10-19.

- Ortiz, P. (2004). Sobralia mutisii P. Ortiz, sp. nov. Orquideología, 23(1), 50-53.

- Ortiz, P. y Uribe, C. (2007). Galería de Orquídeas de Colombia. CD Edition. Bogotá, Colombia: Asociación Bogotana Orquideológica.

- Ortiz, P. y Uribe, C. (2014). Orquídeas, tesoro de Colombia (A-D). Editorial Da Vinci Publicidad y Medios \& cía. S. en. C.

- Pereira, H.; Navarro, L. y Santos, I. (2012). Global biodiversity change: the bad, the good, and unknown. Annual Review of Environment and Resources, 37, 25-50.

- Pridgeon, A. (2005). Sobralia Ruiz \& Pav. En Pridgeon, A.M.; Cribb, P.J.; Chase, M.C. y Rasmussen, F.N. (Eds.), Genera orchidacearum (pp. 601-604). Vol. 4. Oxford: Oxford University Press.

- Sarmiento, J. (2007). La familia Orchidaceae en Colombia. Actualidades biológicas, 29(Supl.1), 21-84.

- Simpson, M. (2010). Plant systematics (Second Edition). UU.EE.: Academic Press. 
1. Lic. Pdn. Agropecuaria, M.Sc Biológicas. Grupo de Investigación en Orquídeas, Ecología y Sistemática Vegetal, Universidad Nacional de Colombia sede Palmira. Palmira, Colombia.mmbonillam@unal.edu.co. ORCID: 0000-0002-2054-6815

2. I.A. Grupo de investigación Horizonte Mediático, Universidad de los Llanos. Villavicencio, Colombia. oscaribanhernandez.90@gmail.com. ORCID: 0000-00030149-0235

3. I.A, Est. M.Sc Biológicas. Grupo de Investigación en Orquídeas, Ecología y Sistemática Vegetal, Universidad Nacional de Colombia sede Palmira. Palmira, Colombia.acaguirrem@gmail.com. ORCID: 0000-0002-5908-0362

Para citar este artículo: Bonilla Morales, M.M.; Hernández Castañeda, O.I. y Aguirre Morales, A.C. (2016). Distribución y preferencias climáticas de Sobralia uribei(Orchidaceae): una especie endémica de Santander, Colombia. Revista Luna Azul, 43, 128-144. Recuperado de http://200.21.104.25/lunazul/index.php?option=com_content\&view=article\&id=196 